\title{
Ultrasonography in diagnosis and analysis of chronic pain following anterior open inguinal herniorrhaphy
}

\author{
ZY Qiu ${ }^{1 \dagger} \mathbb{D}, Y$ Chen $^{1 *}$, JX Tang ${ }^{2}$ and L Chen ${ }^{1+}$
}

\begin{abstract}
Background: Chronic pain as a complication following inguinal herniorrhaphy has attracted increasing attention in recent years. There is evidence that the chronic pain seriously affects patients' quality of life. However, there are few imaging studies and diagnostic techniques of the chronic pain. The aim of this study is to explore the etiology and to analysis ultrasonographic imaging description of chronic pain following anterior open inguinal herniorrhaphy.

Methods: One hundred fifty two patients with the chronic pain following anterior open inguinal herniorrhaphy were performed by ultrasonography to identify the main causes of postoperative chronic pain. Positive ultrasonic diagnoses were confirmed to be correct by the pain relieved when the patients underwent re-operation and other clinical operations. Positive diagnoses which appeared simultaneously were grouped for pairwise comparisons.

Results: Two hundred sixteen positive ultrasonic diagnoses, 12 categories of postoperative chronic pain were found. They were encapsulated effusion, scrotal wall edema, testitis, hydrocele testis, restricted motion of spermatic cord at the reconstructed deep inguinal ring, varicocele, scar sutured into pubic tubercle, shrinking mesh, accumulational mesh or mesh plug, recurrent hernia, cyst of spermatic cord and epididymal cyst. In the pairwise comparison groups, encapsulated effusion with scrotal wall edema, varicocele with restricted motion of spermatic cord at the reconstructed deep inguinal ring, and shrinking mesh with recurrent hernia had significant differences in each intragroup comparisons $(P<0.05)$.

Conclusions: Ultrasonography provieds important value in the diagnosis of chronic pain following anterior open inguinal herniorrhaphy. Some positive diagnoses occur simultaneously, which is necessary for doctors to consider comprehensively.
\end{abstract}

Keywords: Ultrasonography, Anterior open inguinal herniorrhaphy, Chronic pain

\section{Background}

It was pointed out in Guidelines for diagnosis and treatment of adult inguinal hernia (2014 edition) [ 1] that almost all cases of inguinal hernia are treated surgically, and anterior open inguinal herniorrhaphy is considered to be the most common surgical procedure. Also, the chronic pain is a late complication of the surgery. In the 1980s, the chronic pain was reported [2-4] to be a rare and occasional postoperative complication, and the

\footnotetext{
* Correspondence: ultrasound_chen@126.com

${ }^{\dagger}$ ZY Qiu and L Chen contributed equally to this work.

'Ultrasound Department, Huadong Hospital Affiliated to Fudan University,

221 West Yanan Road, Shanghai 200041, China

Full list of author information is available at the end of the article
}

assessment of the surgery was limited to the postoperative acute phase. Nevertheless, studies in recent years have found that the incidence of chronic pain following inguinal herniorrhaphy is approximately $54 \%$, which is far higher than that reported previously. Furthermore, up to $50 \%$ of patients experience the chronic pain for more than 1 year, which seriously affects the patients' quality of life [5-7]. The chronic pain has gradually become one of the long-term assessment indicators of the herniorrhaphy. Non-invasive, nonradiative and easily performed, ultrasound technique has been proposed as a valuable imaging method in examining patients before and after the inguinal herniorrhaphy [8].

(c) The Author(s). 2018 Open Access This article is distributed under the terms of the Creative Commons Attribution 4.0 International License (http://creativecommons.org/licenses/by/4.0/), which permits unrestricted use, distribution, and 
The aim of our study was to explore the etiology and analyze positive findings of chronic pain after anterior open inguinal herniorrhaphy, thereby to assist in making treatment protocols in clinics.

\section{Methods \\ Subjects}

We conducted a prospectively, observational study in a single hernia center of Huadong Hospital, between August 2009 and May 2014. 539 patients who felt uncomfortable after their first herniorrhaphy were performed by ultrasonography in our ultrasound department. 152 patients who met the inclusion criteria agreed to participate in this study and signed the informed consent. This study was approved by Fudan University Ethics Committee. No statistical power calculation was conducted prior to the study. The sample size was based on the previous literature experience about the chronic pain review.

The inclusion criteria: the patients had a history of anterior open inguinal herniorrhaphy, during which artificial material was patched in preperitoneal space or the back wall of the inguinal canal was reconstructed by suturing. According to the definition of chronic pain by International Association of the Study of Pain (IASP) [9], cases with "pain lasting for 3 months or more" were included. Persistent chronic pain with or without local swell in the operative region or the ipsilateral scrotum was the clinical symptom of the disease. Sometimes the symptom could be relieved by holding up the scrotum.

The patients had laparoscope totally extraperitoneal repair(TEP) and transabdominal preperitoneal repair(TAPP) were excluded. And the patients younger than 16 years were excluded.

The longest course of postoperative chronic pain in this study was ten years, and the shortest course was 3 months. Among 31 patients who had a history of bilateral herniorrhaphy, 12 patients presented bilateral chronic pain.

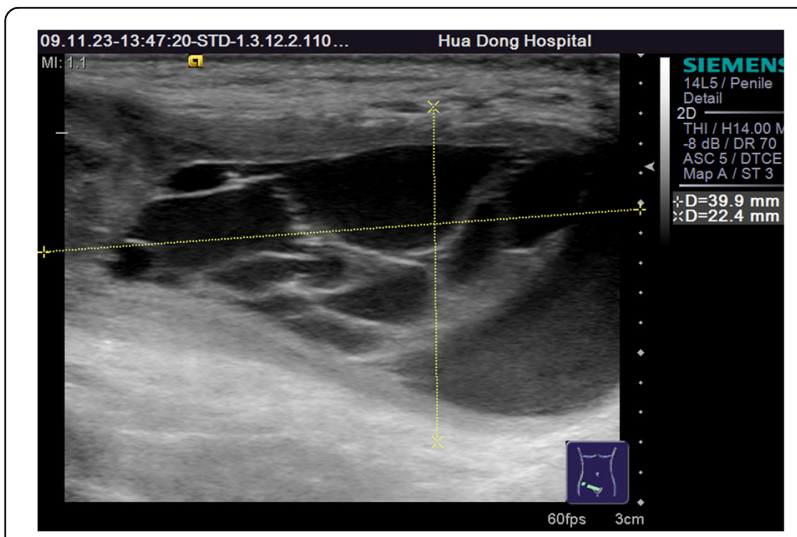

Fig. 1 The seroma showed in the ultrasonography. The multilocular seroma was visible in front of the mesh

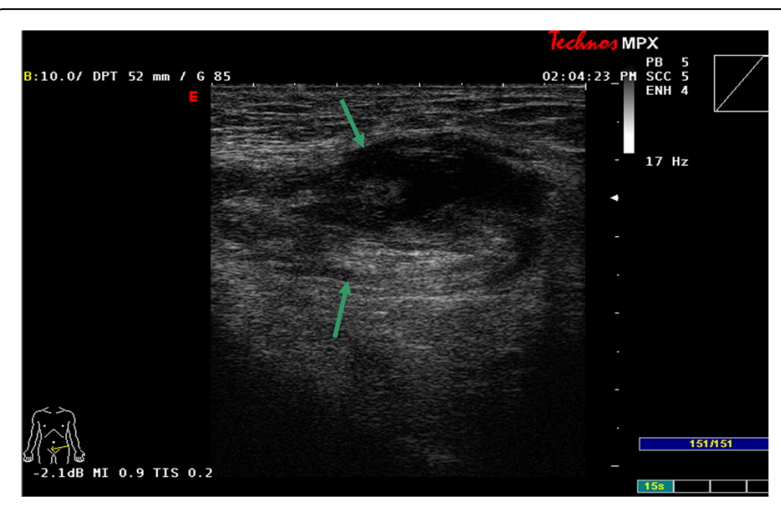

Fig. 2 The hematoma showed in the ultrasonography. Arrows referred to the old hematoma

Ultimately, a total of 164 chronic pain sites met the inclusion criteria, included 157 sites with artificial mesh, 2 sites with mesh plug, and 5 sites with suture repair.

\section{Instruments and methods}

The Siemens ACUSON S2000 ${ }^{\text {mix }}$ color doppler ultrasound instrument, equipped with ultrasonic volume auto-scan(UVAS) and a high-frequency linear transducer ranging from 9 to $12 \mathrm{MHZ}$, were performed to scan the herniorrhaphic incisions and chronic pain sites in patients. The following parameters of UVAS were set: a volume image of $15.4 \mathrm{~cm}$ (length) $\times 16.8 \mathrm{~cm}$ (width) $\times 6.0 \mathrm{~cm}$ (depth) captured with a minimum slice of $0.5 \mathrm{~mm}$ and 250 to 400 images were collected during a single scan. The axial sequence images were automatically transferred to the ultrasonic workstation for data analysis and processing. The patients were in supine position or standing position as needed for ultrasound scanning, with the

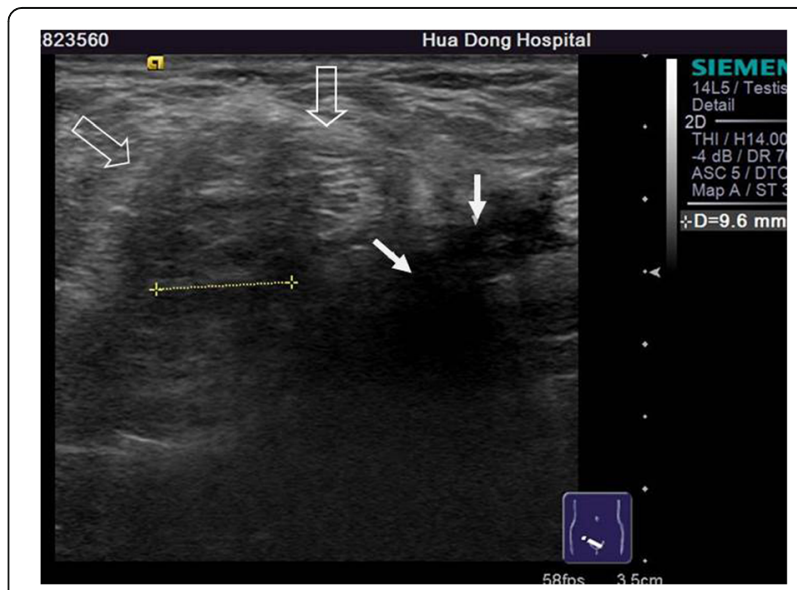

Fig. 3 The recurrent hernia and the shrinking mesh showed in the ultrasonography. Hollow arrows referred to the recurrent hernia and solid arrows referred to the shrinking mesh. The measurement of straight line referred to the diameter of the perito neal defect of the recurrent hernia 


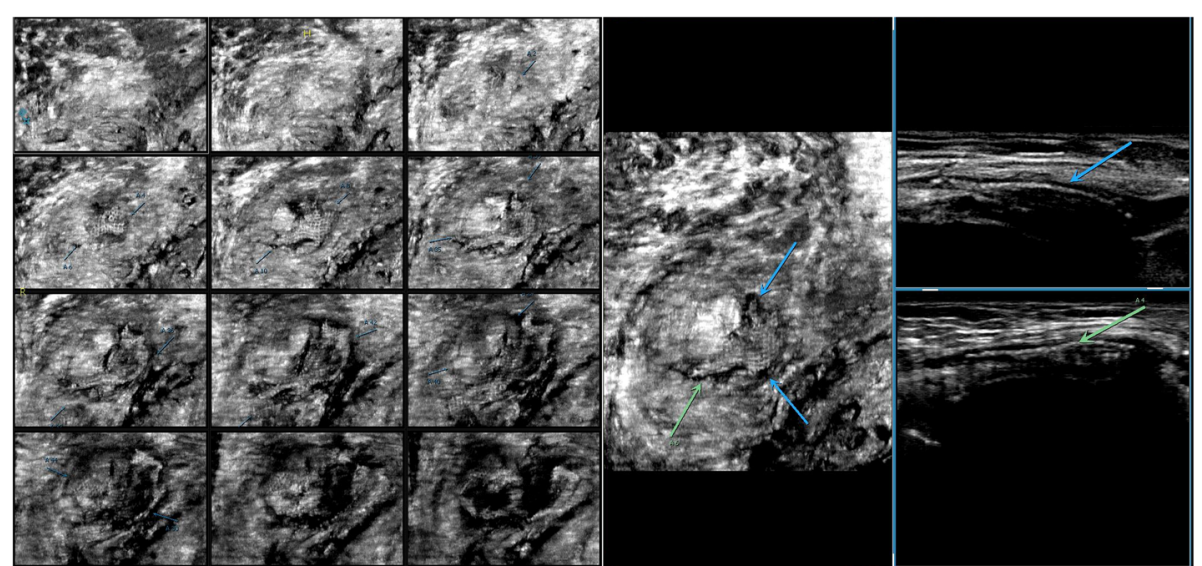

Fig. 4 The shrinking mesh showed in the ultrasonography. The shrinking mesh was showed on coronal consecutive image and 3D display. Arrows referred to the shrinking mesh

scrotums simultaneously scanned in male patients. The location, shape, and spatial structure of the mesh, peritoneum, spermatic cords, etc., were observed, with the goal of achieving ultrasonographic diagnosis of the etiology of the postoperative chronic pain.

All ultrasonographic results were confirmed by the re-operation, the puncture or other clinical operations.

Cases with two or more positive diagnoses were pairwise grouped for multiple comparisons.

\section{Statistical analysis}

Statistical analyses were performed by STATA10 software. Values were presented as mean \pm standard deviation for normally distributed variables. Positive ultrasonic diagnoses in the pairwise groups compared using a chi-square test. Statistical significance threshold was considered $\alpha=0.05$.

\section{Results}

The mean age in the study was 66.10 years $( \pm 13.82 \mathrm{SD})$, ranging from 22 to 93 years old. No statistical difference was observed between genders in 93 men and 59 women $\left(x^{2}=0.35, P>0.05\right)$.

\section{Ultrasonic diagnosis of chronic pain sites following anterior open inguinal herniorrhaphy}

Among 164 chronic pain sites, 121 sites showed positive findings on ultrasonography and no ultrasonic abnormality was found in 43 sites.

Among 121 sites with positive ultrasonic findings, 49 sites had a single positive diagnosis, 49 sites had 2 positive diagnoses and 23 sites had 3 positive diagnoses, which resulted in a total of 216 positive diagnoses: 24 cases of encapsulated effusion (11.1\%), of which 14 cases were confirmed by ultrasound guided aspiration to be seroma (Fig. 1) and 10 cases were confirmed to be hematoma (Fig. 2). 59 cases of recurrent hernia (27.3\%) (Fig. 3), 31 cases of shrinking mesh (14.4\%) (Fig. 4), 5 cases of accumulational patch or mesh plug (2.3\%) (Fig. 5), 2 cases of cyst of spermatic cord $(1.0 \%)$ (Fig. 6) and 1 case of epididymal cyst $(0.5 \%)$ were confirmed surgically. In addition, there appeared to be restricted motion of the spermatic cord at the reconstructed deep inguinal ring in 20 cases(9.2\%)(Fig. 7), including 10 cases of the spermatic cord adhered in the deep inguinal ring, 7 cases of patch compression of the spermatic cord, and 3 cases of reconstructed deep inguinal ring stenosis, all of which were confirmed by adhesiolysis and inguinal neurotomy. Furthermore, ultrasound confirmed 19 cases of the scrotal wall edema (8.8\%), 16 cases of testitis (swelling with increased blood flow) (7.4\%), 16 cases of varicocele(7.4\%)(Fig. 8), and 2 cases of mesh sutured into pubic tubercle(1.0\%), in which the mesh and scar hyperplasia were removed surgically, as well as 5

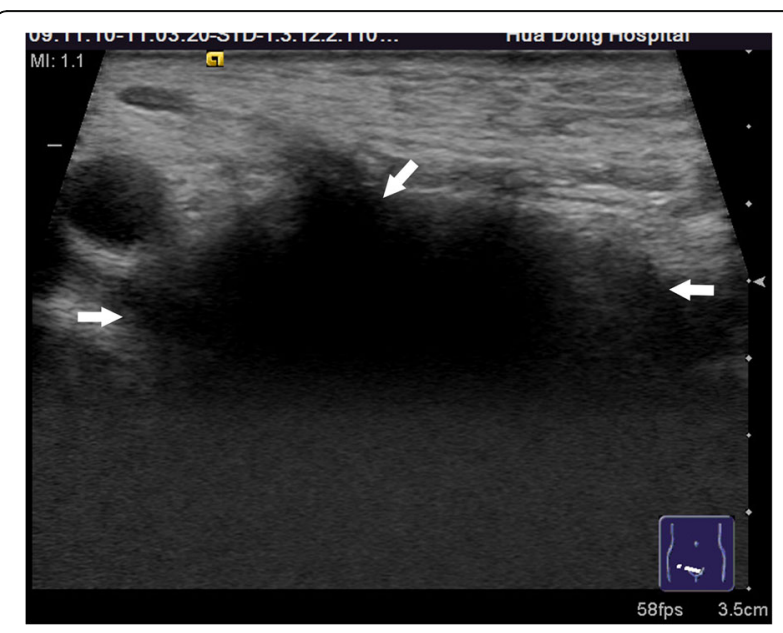

Fig. 5 Accumulational meshes showed in the ultrasonography 


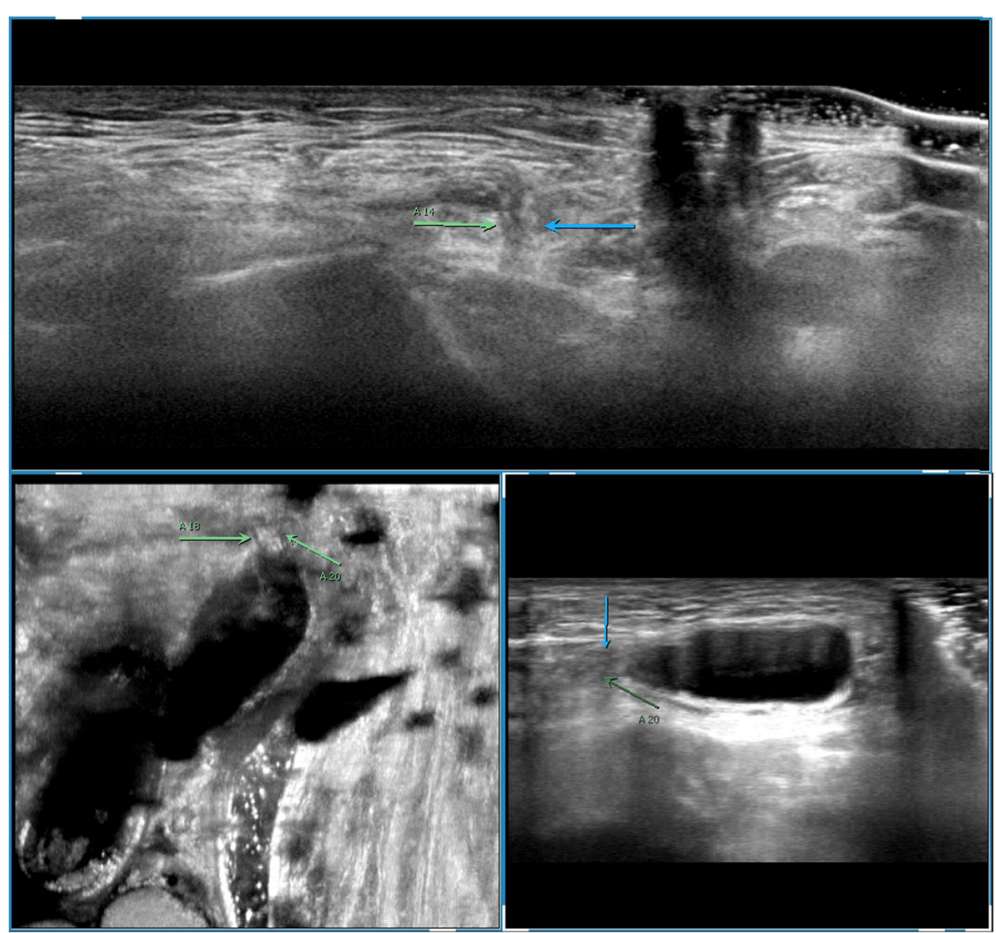

Fig. 6 The cyst of spermatic cord showed in the ultrasonography. Arrows referred to the left spermatic cord

cases of hydrocele testis (2.3\%)(3 cases were confirmed by urological surgery). Ultrasonography revealed 3 cases of local weakness at the reconstructed abdominal wall, which need further confirmation by clinical follow-up.

The pain was relieved or even disappeared when patients had undergone re-operation, puncture, and other clinical operations (such as anti-inflammation therapy and traditional Chinese medicine fomentation).

\section{Comparison of pairwise combination of positive diagnoses}

Ultrasonography revealed that 23 pain sites were found 3 positive diagnoses in each site and 49 pain sites were found 2 positive diagnoses in each site, which resulted in 167 positive diagnoses (Table 1 ).

The intragroup comparisons, grouped according to pairwise combinations of 167 positive diagnoses, showed

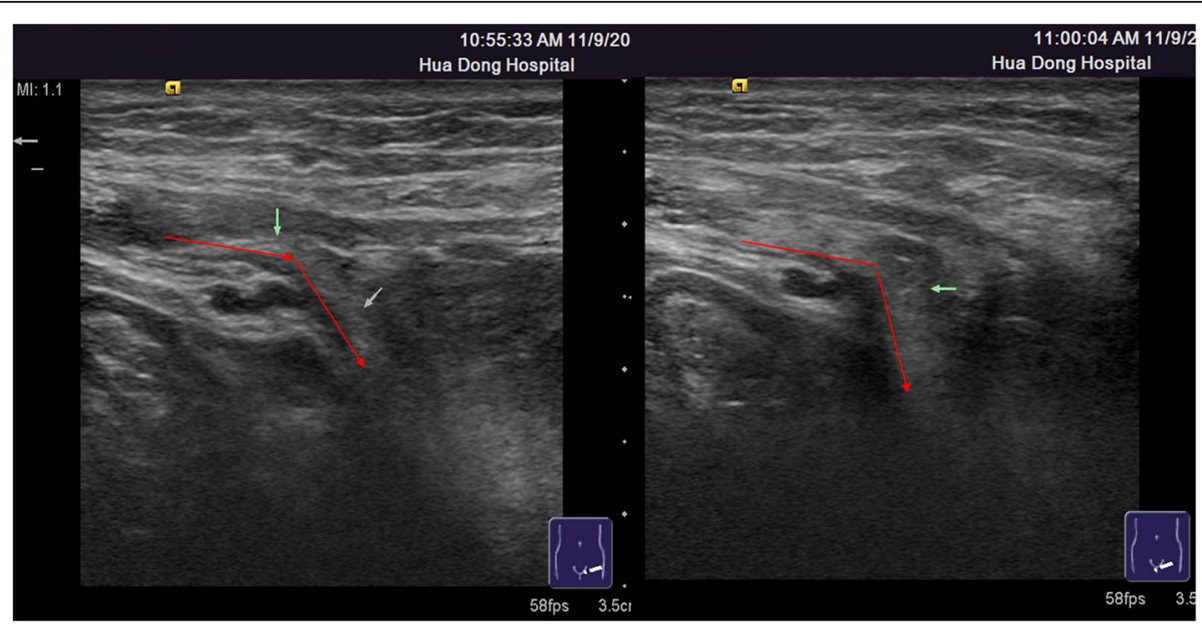

Fig. 7 The spermatic cord showed in the ultrasonography. The spermatic cord was relaxed in supine position(the left graphy). After the patient changed to standing position(the right graphy), the spermatic cord became tight and the bending angle of it was reduced, which indicated that the spermatic cord was adhered in the deep inguinal ring 


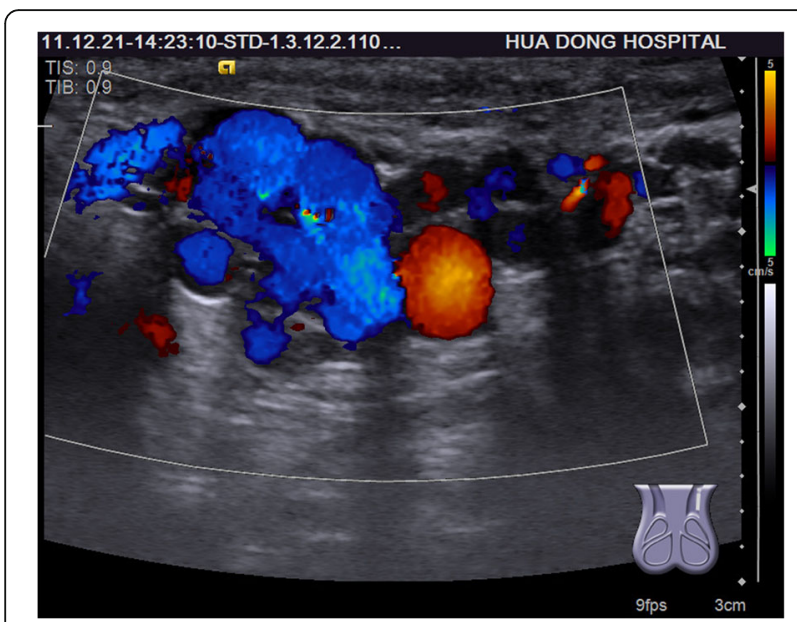

Fig. 8 The varicocele showed in the ultrasonography

that encapsulated effusion synchronized with scrotal wall edema, varicocele synchronized with restricted motion of the spermatic cord at the reconstructed deep inguinal ring, and shrinking mesh synchronized with recurrent hernia had significant differences within the respective groups $(P<0.05)$, which occurred more frequently than other intragroup pairwise combinations.

Meanwhile, other pairwise combinations of positive diagnoses didn't have statistically differences in the intragroup comparisons $(P>0.05)$.
There were 43 cases that postoperative chronic pain occurred, but no insignificant abnormalities were found on ultrasonography. These cases required continued follow-up.

\section{Discussion}

Ultrasonography is a fast, effective and radiation-free examination and suitable for various kinds of positions. UVAS, as a form of advanced ultrasound technology, supplies the coronal consecutive dynamic images on the basis of the two-dimensional images, plays a complementary role to the traditional ultrasound and provides the most intuitive visual plane for clinicians to work out further surgical plans [10]. Ultrasonography can clearly reveal the corrugation of the mesh, the motion of the spermatic cord and the continuity of the peritoneum, which is conducive to diagnosing the cause of the chronic pain after inguinal herniorrhaphy [11]and an effective method of avoiding re-operation in the majority of cases.

There are two main steps of anterior open inguinal herniorrhaphy. One is separation of the hernial sac and the spermatic cord, and the other is to reconstruct the back wall of inguinal canal by suturing or patching an artificial mesh [12]. Ultrasonography shows the morphology and size of the mesh as well as the continuity of the peritoneal peritoneum, which can be used to detect the recurrent hernia. According to the literature, the chronic pain following inguinal herniorrhaphy is mainly

Table 1 Pairwise comparisons of positive diagnoses

\begin{tabular}{|c|c|c|c|c|c|c|c|c|c|c|c|}
\hline & \multicolumn{2}{|c|}{ Encapsulated effusion (1) } & \multicolumn{2}{|c|}{ Varicocele (2) } & \multicolumn{2}{|c|}{ Shrinking mesh (3) } & \multicolumn{2}{|c|}{ Recurrent hernia (4) } & \multirow[t]{2}{*}{ Sum } & \multirow{2}{*}{$\begin{array}{l}x^{2} \\
\text { value }\end{array}$} & \multirow[t]{2}{*}{$P$ value } \\
\hline & Combined & $\begin{array}{l}\text { Un- } \\
\text { combined }\end{array}$ & Combined & $\begin{array}{l}\text { Un- } \\
\text { combined }\end{array}$ & Combined & $\begin{array}{l}\text { Un- } \\
\text { combined }\end{array}$ & Combined & $\begin{array}{l}\text { Un- } \\
\text { combined }\end{array}$ & & & \\
\hline Encapsulated effusion & 23 & 0 & 0 & 23 & 0 & 23 & 5 & 18 & 23 & & \\
\hline Scrotal wall edema & 19 & 0 & 0 & 19 & 0 & 19 & 1 & 18 & 19 & (1) 41.37 & $<0.05$ \\
\hline Testitis & 8 & 2 & 0 & 10 & 0 & 10 & 1 & 9 & 10 & & \\
\hline Hydrocele testis & 2 & 3 & 2 & 3 & 1 & 4 & 0 & 5 & 5 & & \\
\hline $\begin{array}{l}\text { Mesh sutured into } \\
\text { pubic tubercle }\end{array}$ & 0 & 2 & 2 & 0 & 0 & 2 & 0 & 2 & 2 & & \\
\hline $\begin{array}{l}\text { Cyst of spermatic } \\
\text { cord / epididymal } \\
\text { cyst }\end{array}$ & 0 & 2 & 1 & 1 & 0 & 2 & 0 & 2 & 2 & & \\
\hline $\begin{array}{l}\text { Accumulational } \\
\text { mesh }\end{array}$ & 0 & 5 & 1 & 4 & 4 & 1 & 1 & 4 & 5 & & \\
\hline Varicocele & 0 & 16 & 16 & 0 & 4 & 12 & 2 & 14 & 16 & & \\
\hline $\begin{array}{l}\text { Restricted motion } \\
\text { of spermatic cord } \\
\text { at deep inguinal } \\
\text { ring }\end{array}$ & 0 & 20 & 13 & 7 & 8 & 12 & 8 & 12 & 20 & (2) 20.07 & $<0.05$ \\
\hline Shrinking mesh & 0 & 31 & 4 & 27 & 31 & 0 & 23 & 8 & 31 & (4) 13.19 & $<0.05$ \\
\hline Recurrent hernia & 5 & 29 & 2 & 32 & 21 & 13 & 34 & 0 & 34 & (3)7.36 & $<0.05$ \\
\hline Sum & 57 & 110 & 41 & 126 & 69 & 98 & 75 & 92 & 167 & & \\
\hline
\end{tabular}


from somatic, neurological, or visceral sources [13, 14]. Cunningham et al. [15] found that the most common type of postoperative chronic pain was somatic pain, which was mainly associated with pubic tubercle injury caused by suturing the ligament and other tissues into the pubic tubercle. Visceral pain mostly originates from the reproductive system. Ultrasonography can find that the motion of the spermatic cord decreased significantly, the flow of the spermatic vein is obstructed and the spermatic vein is expansive. Ultrasonography can also accurately diagnose the varicocele when the blood flow spectrum of varicose vein was obviously reversed in Valsalva test. Butler et al. [16] reported that the torsion and postoperative stenosis of the spermatic cord caused by scar hyperplasia, which might lead to ejaculatory dysfunction or ejaculation pain. Wantz et al. [13, 17] found that the incidence of ischemic testitis following inguinal herniorrhaphy was $0.61 \%$, which associated with the compression caused by oversized mesh plug or an excessively small patch hole to the spermatic cord. The intraoperative damage or the ligation of spermatic arteries and veins resulted in the obstruction of venous flux and the ischemia of the testis.

Be careful to avoid the sensory nerves when the mesh is being sewed. Neuropathic pain may be due to the inguinal or genitofemoral nerves injury [18]. Ultrasonography has limitation in scanning the nerve branches. This might be the reason for the 43 cases in this study in which postoperative chronic pain was present but no significant abnormalities were apparent on ultrasonography. Caliskan et al. [19] sought the causes of pain by mean of relieving the pain by inguinal neurotomy. Wijsmuller et al. [20] determined the possibly damaged nerves through intraoperative identification and protection of the ilioinguinal nerve and genitofemoral nerve genital branch. Surgical nerve injury might cause the formation of neuromas or complete transection of nerve trunk, while postoperative adhesion and local inflammation might cause scars implanting into the nerve [21, 22].

Therefore, following guidelines, standard operation and normative process are the key to reduce the chronic pain and the other postoperative complications after inguinal herniorrhaphy.

\section{Conclusions}

Both UVAS and traditional ultrasonography have important value in the diagnosis of the chronic pain following anterior open inguinal herniorrhaphy. Some causes of the chronic pain always occur simultaneously which should be comprehensively considered by clinical doctors. Therefore, it is necessary to conside ultrasound technology as an objective basis for the assessment of postoperative chronic pain.
Abbreviation

UVAS: Ultrasonic volume auto-scan

\begin{abstract}
Acknowledgements
We thank Yun Pang who helped us to collect and archived the ultrasonic image data. I am also indebted to Professor Shaojie Li who provided the surgical technical and theoretical guidance for me. My deepest gratitude goes to Professor Jianxiong Tang and Yue Chen for their constant encouragements and illuminating instructions.
\end{abstract}

\section{Funding}

This work was supported by grants for a 2015 Clinical Capacity Building Project for Assistant Departments of the Shanghai Municipal Shenkang Hospital Development Center (project no. SHDC22015013, Chen L).

\section{Availability of data and materials}

We declared that all data and ultrasonic images described in the manuscript, including all relevant raw data, will be freely available to any scientist wishing to use them for non-commercial purposes, without breaching participant confidentiality.

\section{Study design}

All patients in the study who met the inclusion criteria were performed by ultrasonography to identify the main causes of the postoperative chronic pain. Positive ultrasonic diagnoses were confirmed to be correct by the pain relieved or disappeared when the patients had undergone re-operation, puncture, and other clinical operations. Since some positive diagnoses occurred at the same time, we combined these results pairwise with a list. The incidences of pairwise diagnoses were compared in different positive diagnose group. Based on meaningful statistical results, we discussed the advantages and disadvantages of ultrasonography in diagnosing the postoperative chronic pain.

\section{Authors' contributions}

ZQ carried out ultrasonic examinations and diagnosis, performed the statistical analysis and drafted the manuscript. YC and JT participated in the design of the study. LC participated in coordination and helped to draft the manuscript. All authors read and approved the final manuscript.

\section{Ethics approval and consent to participate}

All patients had written the informed consent in which the purpose about this study, the technical information about the ultrasound examination, the research process and the right of the participants were described clearly.

They had been given the opportunity to ask questions about this study, and they had been answered to their satisfaction. All 152 patients consented to participate in this study.

The ethical implications of this study were adequately described in the application for approval of research protocol. This study was approved by the Fudan University Research Ethics Committee.

\section{Consent for publication}

We declared that written informed consents and any ultrasonic images were obtained from the patients for publication of this study.

\section{Competing interests}

The authors declare that they have no competing interests

\section{Publisher's Note}

Springer Nature remains neutral with regard to jurisdictional claims in published maps and institutional affiliations.

\section{Author details}

'Ultrasound Department, Huadong Hospital Affiliated to Fudan University, 221 West Yanan Road, Shanghai 200041, China. ²Department of General Surgery, Huadong Hospital Affiliated to Fudan University, 221 West Yanan Road, Shanghai 200041, China. 
Received: 15 December 2017 Accepted: 9 May 2018

Published online: 22 May 2018

\section{References}

1. Chinese Society for Hernia and Abdominal Wall Surgery CHCOS. Guidelines for diagnosis and treatment of adult inguinal hernia (2014 edition). Chinese Journal of Surgery. 2014;52:481-3.

2. Mudge $M$, Hughes LE. Incisional hernia: a 10 year prospective study of incidence and attitudes. Br J Surg. 1985;72(1):70-1.

3. Luijendijk RW, et al. A comparison of suture repair with mesh repair for incisional hernia. N Engl J Med. 2000;343(6):392-8.

4. Stoppa RE. The treatment of complicated groin and incisional hernias. World J Surg. 1989;13(5):545-54.

5. Poobalan AS, Bruce J, Smith WC, King PM, Krukowski ZH, Chambers WA A review of chronic pain after inguinal Herniorrhaphy. Clin J Pain. 2003; 19(1):48-54

6. Poobalan AS, bruce J, King PM, et al. Chronic pain and quality of life following open inguinal hernia repair. Br J Surg. 2001;88:1122-6.

7. Callesen $\mathrm{T}$, Bech $\mathrm{K}$, Kehlet $\mathrm{H}$. Prospective study of chronic pain after groin hernia repair. Br J Surg. 1999;86:1528-31.

8. Zhiying $\mathrm{Q}$, Jianxiong $\mathrm{T}$. Value of ultrasonography before and after tension-free inguinal hernioplasty. Chinese Journal of General Surgery. 2011;26(2):91-3.

9. International Association for the Study of Pain. Subcommittee on taxonomy. Classification of chronic pain: descriptions of chronic pain syndromes and definitions of pain terms. Pain. 1986;3(Suppl):1-226.

10. Zhiying Q, Yue C, Cai C, et al. Comparison of ultrasonic volume auto-scan and regular ultrasonography for the diagnostic classification of the hernia. Chinese. J Ultrasound Med. 2012;28(5):446-52.

11. Hua T, Lei Z, Hongyan Z, et al. The application of color doppler ultrasonography in diagnosis for the complication of tension-free inguinal hernia repair. Chin J Med Ultrasound (Electronic Edition). 2010;7(9):1483-9.

12. Joachim Conze, Anterior open repair of inguinal hernia in adults. Management of Abdominal Hernias. A.N. Kingsnorth and K.A. LeBlanc (eds.). chapter14.

13. ZibM GJ. Inguinal hernia repair : where to next. ANZ J Surg. 2002;72(8):573.

14. Licheng Z, Junzhong S, Huijun S. Causes and strategies of chronic pain following tension-free herniorrhaphy. Chinese. journal of general practice. 2008;6(8):829-30.

15. Cunningham J, Temple WJ, Mitchell $P$, et al. Cooperative hernia study: pain in the postrepair patient. Ann Surg. 1996;224:598-602.

16. Butler JD, Hershman MJ, Leach A. Painful ejaculation after inguinal hernia repair. J R Soc Med. 1998;91:432-3.

17. Wantz GE. The Canadian repair of inguinal hernia.//Nghus LM, Condon RE. Hernia. 3rd ed. Philadelphia: JB Lippincott Co,1989:236-252.

18. Wijsmuller AR, Lange JFM, Kleinrensink GJ, et al. Nerve-identifying inguinal hernia repair: a surgical anatomical study. World J Surg. 2007;31(2):414-20.

19. Caliskan $\mathrm{K}$, et al. A method for the reduction of chronic pain after tensionfree repair of inguinal hernia: iliohypogastric neurectomy and subcutaneous transposition of the spermatic cord. Hernia. 2010;14(1):51-5.

20. Wijsmuller $A R$, van Veen $R N$, Bosch $J \mathrm{~L}$, et al. Nerve management during open hernia repair. Br J Surg. 2007;94:17-22.

21. Bower $\mathrm{S}$, Moore BB, Weiss SM. Neuralgia after inguinal hernia repair. Am Surg. 1996;62:664-7.

22. Condon RE. Groin pain after hernia repair. Ann Surg. 2001;233(1):8.

\section{Ready to submit your research? Choose BMC and benefit from:}

- fast, convenient online submission

- thorough peer review by experienced researchers in your field

- rapid publication on acceptance

- support for research data, including large and complex data types

- gold Open Access which fosters wider collaboration and increased citations

- maximum visibility for your research: over $100 \mathrm{M}$ website views per year

At BMC, research is always in progress.

Learn more biomedcentral.com/submissions 